Acta Crystallographica Section D

Biological

Crystallography

ISSN 1399-0047

Cathleen Zeymer,* Thomas R. M. Barends, Nicolas D. Werbeck, Ilme Schlichting and Jochen Reinstein*

Department of Biomolecular Mechanisms, Max Planck Institute for Medical Research, Jahnstrasse 29, 69120 Heidelberg, Germany

Correspondence e-mail:

cathleen.zeymer@mpimf-heidelberg.mpg.de, jochen.reinstein@mpimf-heidelberg.mpg.de

\section{Elements in nucleotide sensing and hydrolysis of the AAA+ disaggregation machine ClpB: a structure- based mechanistic dissection of a molecular motor}

Received 17 July 2013

Accepted 7 November 2013

PDB references: ClpB NBD2, wild type, nucleotide-free, 4lj4; wild type, complex with ADP, 4lj5; wild type, complex with AMPPCP, 4lj6; K601Q, complex with MANTdADP, 4lj7; R621Q, complex with ADP, 4lj8; R621Q, complex with AMPPCP, 4lj9; R621Q, complex with AMPPCP and guanidinium chloride, 4 lja 
parallel $\beta$-sheet surrounded by five $\alpha$-helices, and a C-terminal helical bundle. A unifying feature that is shared by all AAA+ proteins, but is also more generally found amongst various families of ATPases and GTPases, is the phosphate-binding loop (P-loop) or Walker A motif characterized by the consensus sequence GXXXXGKT/S (Walker et al., 1982; Saraste et al., 1990). The P-loop residues form interactions with the phosphates of the bound nucleotide. In particular, the highly conserved Walker A lysine was found to be absolutely essential for nucleotide binding and catalysis. The second common feature in AAA+ proteins is the Walker B motif $h h h h \mathrm{DE}$, where $h$ is a hydrophobic amino acid (Walker et al., 1982). The highly conserved Walker B glutamate is believed to activate the attacking water molecule during ATP hydrolysis. $\mathrm{AAA}+$ proteins are active as oligomers, with the catalytic site being located at the interface between two NBD subunits in the oligomer. Highly conserved arginine residues, termed arginine fingers, are located in this interface and are believed to contact the nucleotide of the neighbouring subunit (Ogura et al., 2004). Furthermore, conserved sensor motifs have been identified and proposed to be involved in nucleotide-state sensing, allosteric communication or discrimination between the ADP and ATP states, which is a prerequisite for a functioning ATPase motor (Guenther et al., 1997). The sensor 1 motif contains a conserved polar residue (N/T) that is essential for ATP hydrolysis. The sensor 2 motif (GAR) located on the C-terminal helical bundle contains a conserved arginine residue that can interact directly with the nucleotide. A model of the typical AAA+ catalytic site with the essential residues in their active conformation is depicted in Fig. 1.

The AAA+ disaggregation machines Hsp104 and ClpB possess two NBDs per monomer, thus forming double-ring structures with 12 potential ATPase sites in the active hexameric complex. In order to dissect interfering processes in the oligomeric context of the full-length protein, the $\mathrm{N}$-terminal nucleotidebinding domain (NBD1) and the C-terminal nucleotide-binding domain (NBD2) of ClpB from Thermus thermophilus were studied as separately expressed constructs with regard to nucleotide binding, oligomerization and ATPase activity (Beinker et al., 2005; Werbeck et al., 2009; Zeymer et al., 2013). On the basis of this detailed analysis, we are now able to tackle open mechanistic questions concerning nucleotide-state sensing and the conversion of chemical energy from ATP hydrolysis into mechanical motion. We determined high-resolution X-ray structures in different nucleotide states for one of the motor subunits, namely NBD2, of $\mathrm{ClpB}$ and combined the structural analysis with nucleotide-binding kinetics studies. Our results provide a better understanding of how the ATPase cycle of the molecular motor is regulated and modulated, which is essential in order to gain further insights into the functioning of the complete disaggregation machine.

\section{Materials and methods}

\subsection{Construct design and mutagenesis}

The design of the ClpB NBD2 construct, comprising amino acids 520-854 of ClpB from T. thermophilus, has been described previously (Beinker et al., 2005). The active-site mutations K601Q, R621Q, N709A and R806A were introduced by PCR according to the QuikChange protocol (Agilent Technologies, Santa Clara, California, USA). DNA sequencing was performed by Eurofins MGW Operon (Ebersberg, Germany).

\subsection{Protein expression and purification}

All ClpB NBD2 variants were expressed recombinantly in Escherichia coli BL21 (DE3) RIL cells and purified as described previously (Beinker et al., 2005; Werbeck et al., 2009). The proteins were stored in buffer $A(50 \mathrm{~m} M$ Tris- $\mathrm{HCl}$ $\mathrm{pH} 7.5,50 \mathrm{~m} M \mathrm{KCl}, 5 \mathrm{~m} M \mathrm{MgCl}_{2}, 2 \mathrm{~m} M$ EDTA).

\subsection{Crystallization and structure determination}

Crystals of all ClpB NBD2 variants were grown in a hanging-drop vapour-diffusion setup at $20^{\circ} \mathrm{C}$. The protein

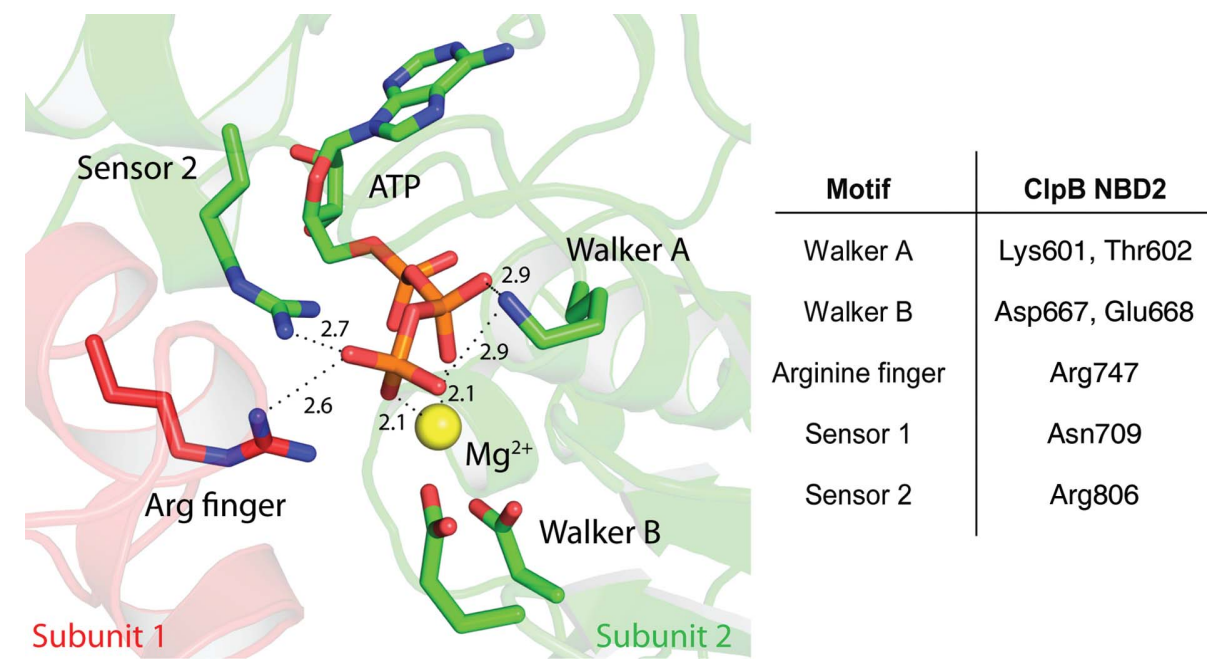

Figure 1

Model of a typical AAA + catalytic site in its active conformation. The catalytic site is located at the interface between two neighbouring subunits (red and green) in the oligomeric AAA+ complex. The Walker A lysine, which is crucial for nucleotide binding and catalysis, interacts directly with the phosphates of the bound nucleotide. The essential $\mathrm{Mg}^{2+}$ ion (yellow) is bound between the $\beta$ - and $\gamma$-phosphates of ATP. The Walker B aspartate coordinates the $\mathrm{Mg}^{2+}$ ion (either directly or via the water molecules of the hydration shell). The Walker B glutamate is positioned such that it can activate the attacking water molecule. A conserved catalytically essential arginine residue from the neighbouring subunit (arginine finger) contacts the bound nucleotide. Conserved sensor-motif residues are in close proximity and are involved in nucleotide-state sensing and allosteric communication. This figure was generated based on the structure of the E. coli clamp loader (PDB entry 3glf; Simonetta et al., 2009). Distances are given in $\AA$. Corresponding residues in ClpB NBD2 are listed next to the model. 
Table 1

Crystallographic data-collection and refinement statistics.

Values in parentheses are for the highest resolution shell. n.a., not applicable.

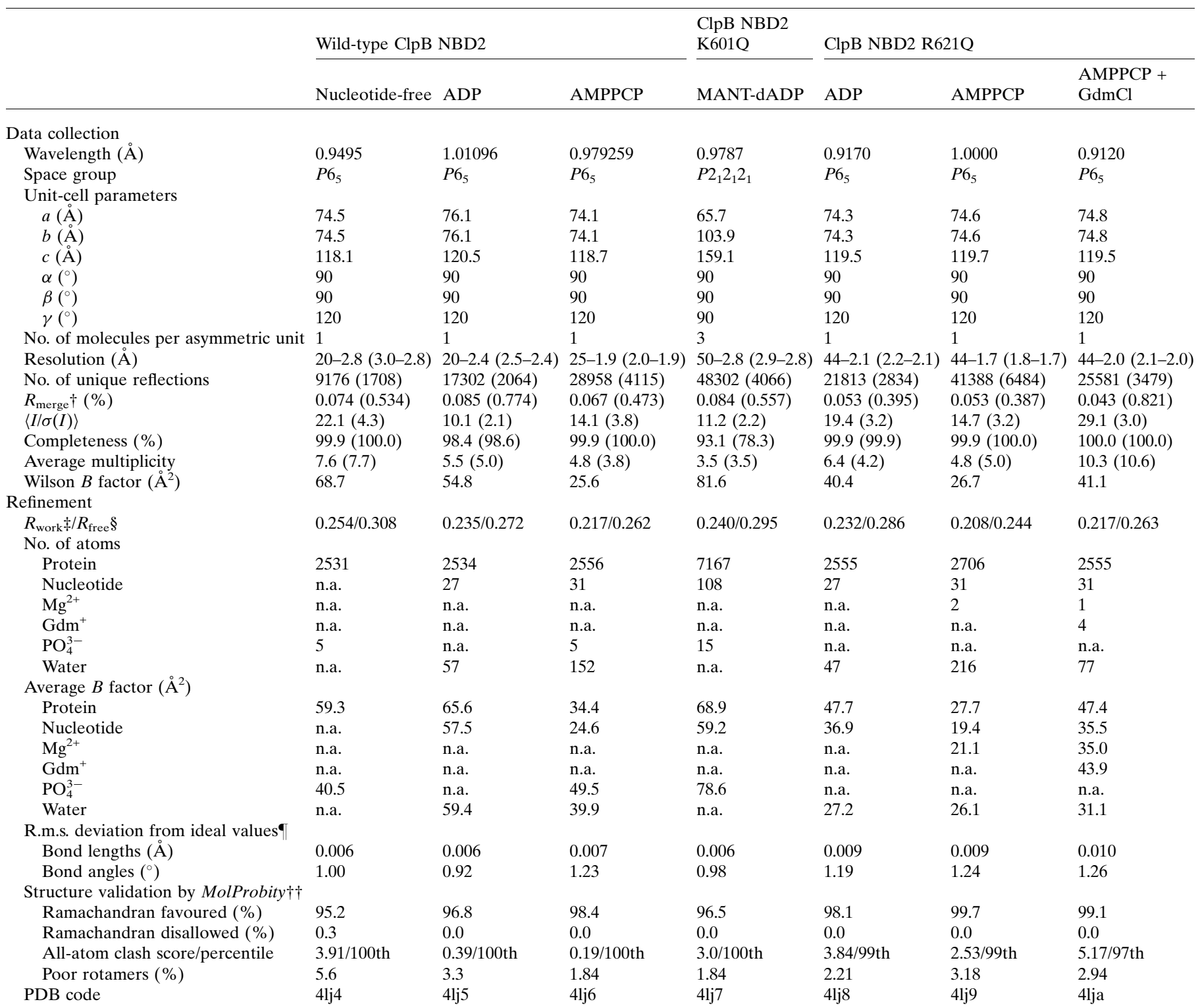

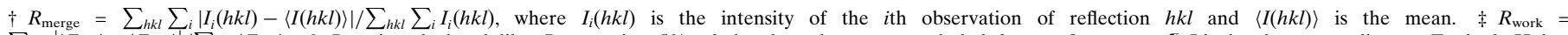

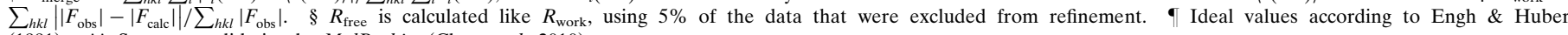
(1991). †† Structure validation by MolProbity (Chen et al., 2010).

solution $\left(\sim 8 \mathrm{mg} \mathrm{ml}^{-1}\right.$ in buffer $A$ ) was either nucleotide-free or was supplemented with $2 \mathrm{~m} M$ ADP, $2 \mathrm{~m} M$ MANT-dADP or $2 \mathrm{~m} M$ AMPPCP and was mixed in a 1:1 ratio with the reservoir solutions. Crystals of wild-type ClpB NBD2 (space group $\left.P 6_{5}\right)$ in the nucleotide-free state and in complex with ADP or with AMPPCP grew in $0.2 M$ sodium citrate, $0.1 M$ HEPES$\mathrm{NaOH}$ pH 7.0, 20\% 2-propanol. Adding 15\%(v/v) PEG 400 afforded sufficient cryoprotection for flash-cooling in liquid nitrogen. The reservoir solution for the crystallization of $\mathrm{ClpB}$ NBD2 K601Q in complex with MANT-dADP (space group $\left.P 2{ }_{1} 2_{1} 2_{1}\right)$ consisted of $0.1 M$ HEPES-NaOH pH 7.5, $12 \%$ PEG $6000,50 \mathrm{mM} \mathrm{MgCl}$. The cryoprotectant solution was additionally supplemented with $25 \%$ glycerol and $2 \mathrm{~m} M$ MANT-
dADP. Crystals of ClpB NBD2 R621Q in complex with ADP or AMPPCP (space group $P 6_{5}$ ) grew in $0.1 M$ Tris- $\mathrm{HCl} \mathrm{pH} 7.5$, 15-20\% 2-propanol, $10-100 \mathrm{~m} M \mathrm{MgCl}_{2}$. The cryoprotectant solution was additionally supplemented with $15 \%$ PEG 400 and $2 \mathrm{~m} M$ of the respective nucleotide. Crystals were passed quickly through the cryoprotectant solutions and were flashcooled in liquid nitrogen. Where indicated, crystals of $\mathrm{ClpB}$ NBD2 R621Q were soaked in cryoprotectant solution supplemented with $100 \mathrm{~m} M \mathrm{GdmCl}$ for $60 \mathrm{~min}$ prior to cooling. Diffraction data collection was performed on beamline X10SA at the Swiss Light Source (Villigen, Switzerland) with the crystals maintained at $100 \mathrm{~K}$. The $X D S$ program package was used for data processing (Kabsch, 2010). Phasing 
of the ADP-bound wild-type structure was performed by molecular replacement using CNS with residues 520-854 of the full-length ClpB structure (PDB entry 1qvr; Lee et al., 2003) as the search model. Patterson correlation refinement (Brünger, 1990) was required owing to the different domain arrangement of the search model. Subsequent structures were solved using Phaser (McCoy et al., 2007). Model building and refinement was performed in iterative cycles using Xfit (McRee, 1999) or Coot (Emsley \& Cowtan, 2004) and REFMAC (Murshudov et al., 2011) including TLS refinement (Winn et al., 2001), respectively. The model quality was validated with MolProbity (Chen et al., 2010). Structure illustrations were generated using $P y M O L$ (Schrödinger). The coordinates and structure factors have been deposited in the Protein Data Bank (PDB entries 4lj4, 4lj5, 4lj6, 4lj7, 4lj8, 4lj9 and 4lja).

\subsection{Stopped-flow experiments and global fitting analysis of nucleotide-binding data}

Nucleotide-binding kinetics measurements of ClpB NBD2 variants were performed using a BioLogic SFM-400 stoppedflow instrument (BioLogic Science Instruments, Claix, France) in single mixing configuration at $25^{\circ} \mathrm{C}$, essentially as described before for the wild-type protein (Werbeck et al., 2009). The fluorescently labelled nucleotides MANT-dADP and MANTdATP were purchased from BIOLOG (Bremen, Germany). The excitation wavelength was $296 \mathrm{~nm}$ and the fluorescence signal was observed using a $400 \mathrm{~nm}$ longpass filter (400FG0325, LOT Oriel Group). This setup was used to selectively excite protein-bound MANT-nucleotides via fluorescence resonance energy transfer (FRET) from the initially excited tryptophan residues of the protein. Measurements were

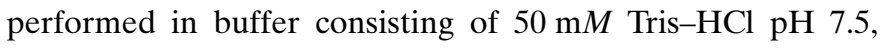
$200 \mathrm{~m} M \mathrm{KCl}, 5 \mathrm{~m} M \mathrm{MgCl}_{2}, 2 \mathrm{~m} M$ EDTA. A set of direct mixing, displacement and competition experiments was performed to determine the association rate constants $k_{\text {on }}$ and the dissociation rate constants $k_{\text {off }}$ for both labelled nucleotides (MANT-dADP and MANT-dATP) and unlabelled nucleotides (ADP and ATP). The nucleotide-binding affinity $K_{\mathrm{d}}$ was calculated from the ratio $k_{\text {off }} / k_{\mathrm{on}}$. ATP-binding experiments were performed in the presence of $0.01 \mathrm{mg} \mathrm{m}^{-1}$ pyruvate kinase and $200 \mu M$ phosphoenol pyruvate as an ATP-regenerating system (MANT-nucleotides are unaffected by pyruvate kinase). Firstly, direct mixing experiments were performed in which $2 \mu M$ ClpB NBD2 was mixed with different concentrations $(5-25 \mu M)$ of MANT-dADP or MANT-dATP. In displacement experiments, $2 \mu M$ ClpB NBD2 was incubated with $5 \mu M$ MANT-dADP or MANTdATP and then mixed with different concentrations of ADP (0.5-5 mM). In competition experiments, ClpB NBD2 was mixed simultaneously with both MANT-dADP and unlabelled nucleotide (ADP or ATP), with final mixing concentrations of $0.5 \mu M$ protein, $2.5 \mu M$ MANT-dADP and 2.5-100 $\mu M$ ADP or 25-300 $\mu M$ ATP. Additional experimental data were needed to unambiguously determine the ATP-binding parameters. We therefore performed another displacement experiment in which $1 \mu M$ ClpB NBD2 was incubated with $200 \mu M$ ATP (in the presence of phosphoenol pyruvate and pyruvate kinase as an ATP-regenerating system) and then mixed with 10-100 $\mu M$ MANT-dADP. The different experimental data sets were combined in a global fitting analysis using the program DYNAFIT (Kuzmic, 1996). The kinetic model given in the scheme below (where $\mathrm{Nt}=\mathrm{ADP}$ or ATP) was applied for the fitting procedure, which utilizes numerical integration of the corresponding differential equations and nonlinear least-squares minimization.

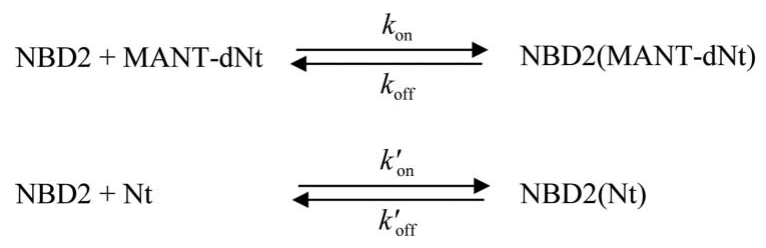

The rate constants were treated as global parameters that were shared by all data sets included; the response coefficients that reflect the amplitude information were treated as semiglobal parameters shared among one experimental series.

\subsection{Steady-state ATPase assay}

The steady-state ATPase activity of ClpB NBD2 variants was measured in a coupled colorimetric assay essentially as described previously (Schlee et al., 2001; Beinker et al., 2005). The measurements were performed at $25^{\circ} \mathrm{C}$ using a Jasco V-650 spectrophotometer (Jasco Germany GmbH). $10 \mu M$ protein was incubated in assay buffer $(50 \mathrm{~m} M$ Tris- $\mathrm{HCl} \mathrm{pH}$ 7.5, $100 \mathrm{~m} M \mathrm{KCl}, 2 \mathrm{~m} M$ EDTA, $2 \mathrm{~m} M$ DTE, $0.8 \mathrm{~m} M$ phosphoenolpyruvate, $0.4 \mathrm{~m} M \mathrm{NADH}, 0.1 \mathrm{~g} \mathrm{l}^{-1} \mathrm{BSA}, 4 \mathrm{U} \mathrm{ml}^{-1}$ pyruvate kinase, $6 \mathrm{U} \mathrm{ml}^{-1}$ lactate dehydrogenase, $5 \mathrm{mM}$ $\mathrm{MgCl}_{2}$ ). The reaction was started by adding $1 \mathrm{~m} M$ ATP. The decrease in NADH absorption was monitored at $340 \mathrm{~nm}$ over time and the maximum slope was used to calculate the ATPase turnover rate per molecule.

\section{Results and discussion}

3.1. High-resolution structures of ClpB NBD2 in different nucleotide states reveal an inactive, open conformation of the catalytic site

In order to understand the mechanism behind the force generation upon ATP hydrolysis in the disaggregation machine $\mathrm{ClpB}$, we aimed at obtaining high-resolution crystal structures of one of its two nucleotide-binding domains, NBD2, in different nucleotide states. We sought to determine structural differences between snapshots of different stages during the ATPase cycle in order to reveal the essential conformational changes that drive this molecular motor. We therefore crystallized ClpB NBD2 from T. thermophilus under nucleotide-free conditions as well as in the presence of ADP and the nonhydrolysable ATP analogue AMPPCP. Crystallization experiments using 2-propanol as the precipitant yielded well diffracting diamond-shaped crystals for all three states. Structures were determined by molecular replacement 


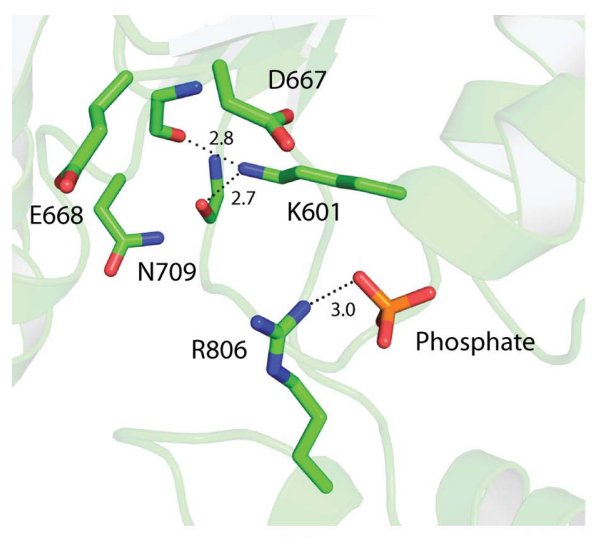

$(a)$

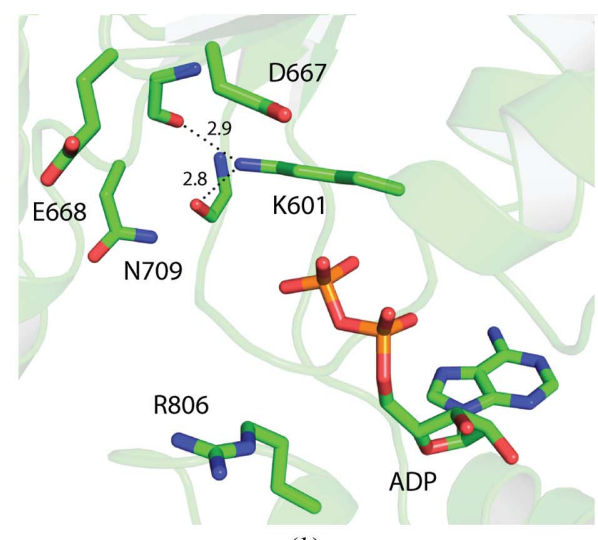

(b)

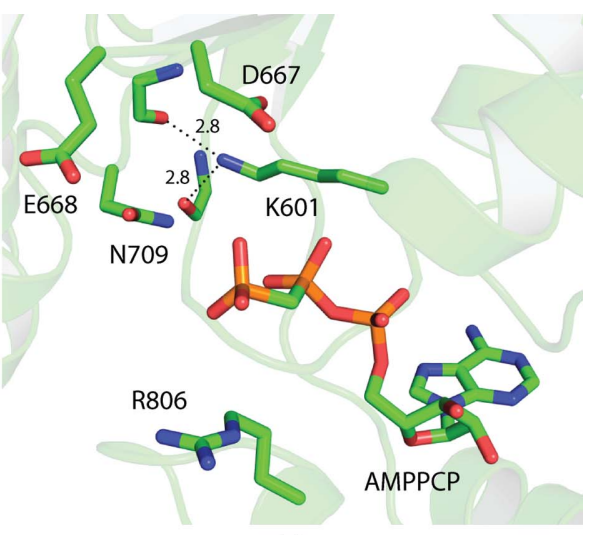

(c)

Figure 2

Crystal structures of wild-type ClpB NBD2 in the absence of nucleotide $(a)$ and in complex with ADP $(b)$ and the nonhydrolysable ATP analogue AMPPCP $(c)$. The three structures are highly similar, showing an open and inactive arrangement of the catalytic site, independent of the nucleotide state. Strikingly, the Walker A lysine Lys601 is present in a fully stretched conformation, pointing away from the nucleotide and forming hydrogen bonds with the backbone carbonyl groups of Gly595 and Ser708. This leads to an unfavourable positioning of the catalytically essential residues of the Walker B motif (Asp667 and Glu668) and the sensor 2 motif (Asn709). The sensor 2 residue Arg806 is captured by a crystal contact with the neighbouring NBD2 molecule in both the ADP-bound and AMPPCP-bound forms. In the nucleotide-free state Arg806 interacts with the inorganic phosphate ion bound in the catalytic site. Distances are given in $\AA$.

to resolutions of $2.8 \AA$ (nucleotide-free), $2.4 \AA$ (ADP) and $1.9 \AA$ (AMPPCP) (Table 1). The ClpB NBD2 molecules exhibit a helical arrangement with sixfold symmetry owing to the hexagonal screw axis of the crystal lattice. Therefore, the interface between neighbouring NBD2 molecules is slightly shifted compared with a planar hexameric complex, which to date could only be modelled based on electron-microscopy data (Lee et al., 2007; Biter et al., 2012) and not observed in $\mathrm{X}$-ray structures of $\mathrm{ClpB}$. In our structures, the proposed arginine finger Arg747 located in this interface is about $11 \AA$ away from the nucleotide bound to the neighbouring NBD2 molecule. The overall fold of our structures is very similar to recently published structures of ClpB NBD2 (Biter et al., 2012) and the corresponding part of the structure of fulllength ClpB (Lee et al., 2003). However, we observed a deviation in the positioning of the helix-loop-helix motif comprising residues $710-740$, which is most likely to be caused by alternative crystal contacts in our crystal form. Most importantly, our structures of the nucleotide-free and AMPPCP-bound states are of significantly higher resolution than those available previously, allowing a more detailed structural analysis.

We focused on analyzing the arrangement of the mechanistically relevant residues in the catalytic site (Fig. 2). The nucleotide-bound structures contained one molecule of ADP or AMPPCP positioned by the highly conserved hydrogenbonding network consisting of nucleobase interactions as well as hydrogen bonds between the phosphate groups and the P-loop residues. The nucleotide-free structure contained a phosphate ion bound at the same position as the $\alpha$-phosphate in the nucleotide-bound structures. This single phosphate ion forms a salt bridge $(d=3.0 \AA$ ) with the positively charged side chain of the sensor 2 residue $\operatorname{Arg} 806$, which is not observed in the nucleotide-bound states, in which Arg806 is involved in a crystal contact with Glu520 of a neighbouring molecule. Apart from this, the structures of the different nucleotide states are highly similar. Strikingly, in all ClpB NBD2 structures determined to date the side chain of the Walker A lysine Lys601, which is crucial for nucleotide binding and hydrolysis, is present in a completely stretched conformation that prevents the expected interaction with the $\beta / \gamma$-phosphates of the bound nucleotide (Fig. 2). Instead, the stretched Lys601 side chain forms hydrogen bonds to the carbonyl backbone groups of Gly595 and Ser708. These unusual interactions directly influence both the conformation of the P-loop (Gly595-Thr602) and the essential sensor 1 motif (Asn709). They furthermore stabilize an open, inactive conformation of the catalytic site in which the Walker B motif consisting of Asp667 and Glu668, which is believed to activate the attacking water molecule for ATP hydrolysis, cannot come close enough to fulfil its task and therefore rests in an inactive position. The second remarkable feature observed in all ClpB NBD2 structures is the lack of the essential $\mathrm{Mg}^{2+}$ ion usually coordinated by the phosphates of the bound nucleotide. Despite extensive efforts including cocrystallization with up to $100 \mathrm{mM} \mathrm{MgCl}$, it was not possible to incorporate the crucial $\mathrm{Mg}^{2+}$ ion into the nucleotide-bound structures of wild-type ClpB NBD2. This further supports the hypothesis that the catalytic site arrangements obtained to date reflect an inactive resting state with regard to ATP hydrolysis of ClpB NBD2. Importantly, this inactive picture characterized by a stretched Walker A lysine conformation and the absence of $\mathrm{Mg}^{2+}$ from the catalytic site is not only observed in our structures but also in all other ClpB NBD2 structures published to date, independent of the nucleotide state (Biter et al., 2012; Lee et al., 2003).

\subsection{Implications from a ClpB NBD2 Walker A mutant (K601Q) structure in complex with the fluorescently labelled nucleotide MANT-dADP}

In order to perturb the open, inactive arrangement of the ClpB NBD2 catalytic site, we replaced the Walker A lysine 
with a glutamine. It has previously been shown that this mutation causes severe defects in both nucleotide binding and ATPase activity (Schlee et al., 2001). To crystallize the mutant ClpB variant NBD2 K601Q, we supplemented the crystallization cocktail with the fluorescently labelled nucleotide MANT-dADP, which has been routinely used for nucleotidebinding studies of $\mathrm{ClpB}$ and is known to bind more than 100fold more strongly to NBD2 than unlabelled ADP (Werbeck et al., 2009). This compensated for the loss in binding affinity owing to the Walker A mutation and allowed us to obtain a nucleotide-bound structure of ClpB NBD2 K601Q (Fig. 3).

The complex of ClpB NBD2 K601Q and MANT-dADP crystallized from a condition containing PEG 6000 and yielded a different crystal form to that observed previously for the wild-type protein in conditions containing 2-propanol $\left(P 2_{1} 2_{1} 2_{1}\right.$ instead of $P 6_{5}$ ). The structure was determined by molecular

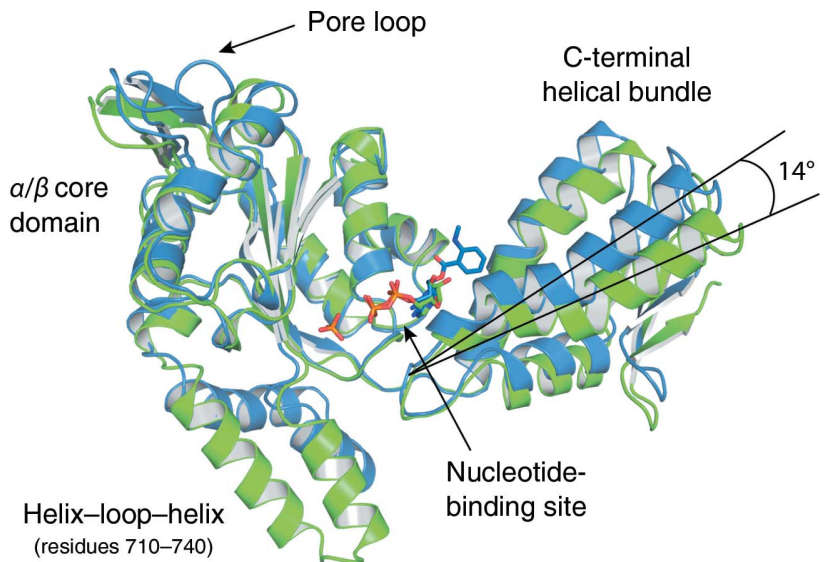

(a)

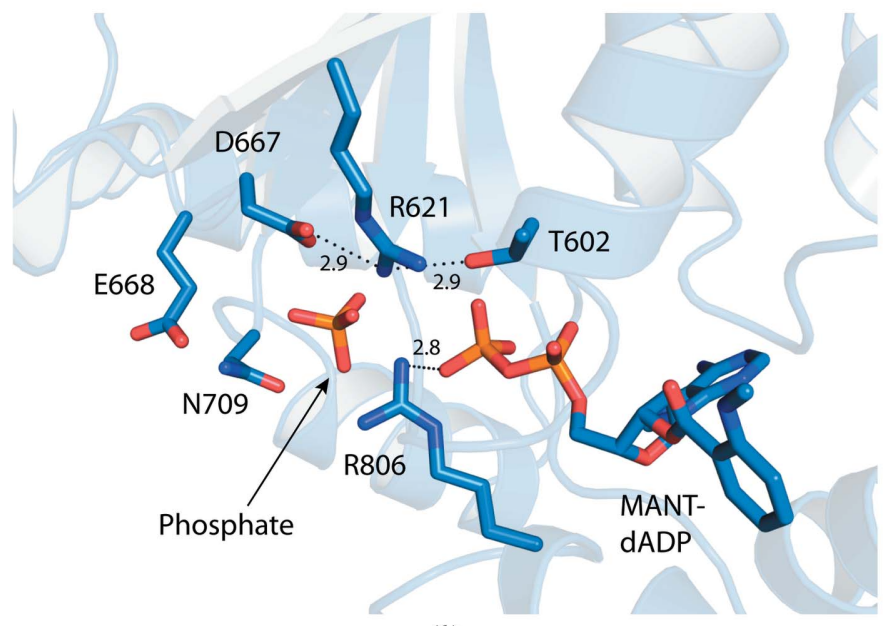

(b) replacement to a resolution of $2.8 \AA$ (Table 1), showing well defined electron density for the previously unstructured poreloop region (residues 640-650). The fluorescently labelled MANT-dADP binds at the same position as the unlabelled nucleotides in the structures obtained previously. The aromatic MANT group attached to the $3^{\prime}-\mathrm{OH}$ of the ribose fits well into a mainly hydrophobic cavity (Fig. 3c), which also explains the preferential binding of 3'-MANT-ADP compared with 2'-MANT-ADP owing to steric restrictions (Werbeck et al. 2009). The catalytic site shows a more closed conformation, characterized by a narrowing of the cleft between the large $\alpha / \beta$ core domain and the C-terminal small domain (Fig. 3a). A domain-motion analysis using DynDom (Hayward \& Lee, 2002) comparing the MANT-dADP-bound form with the ADP-bound form showed that the angle between the domains decreases by $14^{\circ}$. The essential sensor 2 residue $\operatorname{Arg} 806$ located on the C-terminal small domain forms a salt bridge $(d=2.8 \AA)$ with the $\beta$-phosphate of the nucleotide. An additional phosphate ion is present in the active site at a distance of $5 \AA$ from the $\beta$-phosphate of the nucleotide, suggesting that we may have captured an ADP $+\mathrm{P}_{\mathrm{i}}$ (product-bound) state here. However, this structure also does not reveal the active arrangement of the essential Walker B motif that would explain its mode of action. Furthermore, we were again not able to observe the crucial $\mathrm{Mg}^{2+}$ ion in the catalytic site. Interestingly, another highly conserved active-site residue, Arg621, adopts a different conformation to that in the structures described previously. This arginine points towards the nucleotide and is positioned directly between the Walker A

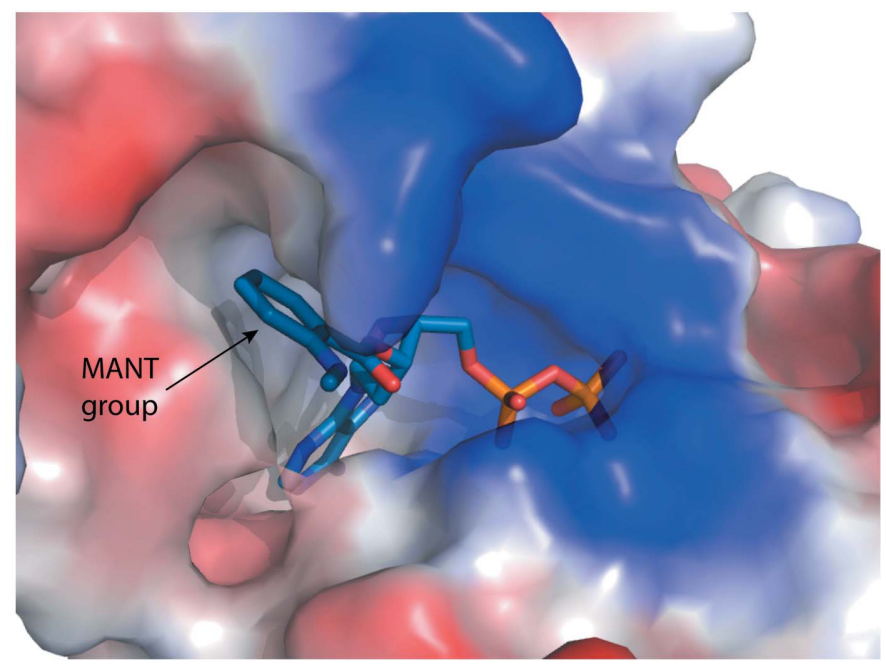

(c)

Figure 3

ClpB NBD2 K601Q in complex with MANT-dADP. (a) The two different crystal forms obtained for ClpB NBD2. Wild-type protein in complex with ADP, space group $P 6_{5}$, is shown in green. Walker A mutant K601Q in complex with MANT-dADP, space group $P 2_{1} 2_{1} 2_{1}$, is shown in blue. The cleft between the core domain and the C-terminal helical bundle is narrowed by $14^{\circ}$ in the structure of ClpB NBD2 K601Q in complex with MANT-dADP, leading to a slightly more closed catalytic site. There is a large deviation of the helix-loop-helix motif (710-740), which is owing to different crystal contacts. Furthermore, we observe clear electron density for the previously unstructured pore-loop region. $(b)$ The catalytic site arrangement of ClpB NBD2 K601Q in complex with MANT-dADP. An inorganic phosphate ion is located $5 \AA$ from the $\beta$-phosphate of the bound nucleotide, suggesting an $\mathrm{ADP}+\mathrm{P}_{\mathrm{i}}$ (product-bound) state. The sensor 2 residue Arg806 interacts with the $\beta$-phosphate of the nucleotide. The conserved residue Arg621 is located in between the Walker A threonine Thr602 and the Walker B aspartate Asp667 at a position where the essential $\mathrm{Mg}^{2+}$ ion is expected to bind. Distances are given in $\AA$. (c) The MANT group of the fluorescently labelled nucleotide MANT-dADP fills a mainly hydrophobic cavity in ClpB NBD2, explaining the higher binding affinity of MANT-dADP compared with the unlabelled nucleotide ADP. The electrostatic surface potential is visualized (red, negative charge; blue, positive charge). 
residue Thr602 and the Walker B residue Asp667, thereby causing an arrangement that might block the incorporation of $\mathrm{Mg}^{2+}$ because it places a positive charge close to where the positively charged $\mathrm{Mg}^{2+}$ ion is expected to bind (Fig. $3 b$ ). With the intention to facilitate the incorporation of $\mathrm{Mg}^{2+}$ and thus obtain an active structure, we next generated the mutant variant ClpB NBD2 R621Q and performed both biochemical characterization and crystallization experiments, which are described in the following sections.

\subsection{The active-site mutations $R 806 \mathrm{~A}$ and $\mathrm{R} 621 \mathrm{Q}$ modify the discrimination between ADP and ATP binding and influence the ATPase activity of ClpB NBD2}

Changes in nucleotide-binding properties owing to activesite mutations provide very useful insights regarding the mechanistic roles of the respective residues in ATP hydrolysis and nucleotide-state sensing. The nucleotide-binding parameters of ClpB NBD2 have been characterized previously using transient kinetics experiments with fluorescently labelled MANT-nucleotides (Werbeck et al., 2009). A set of stopped-flow experiments including direct mixing, displacement and competition experiments in combination with global fitting analysis allowed the determination of the binding parameters for both MANT-labelled as well as unlabelled nucleotides. Interestingly, the binding affinity of ClpB NBD2 for ADP is almost 200-fold higher than for ATP. This strong discrimination is mainly owing to a lower dissociation rate constant $k_{\text {off }}$ for ADP (Werbeck et al., 2009). However, the $\mathrm{X}$-ray structures available to date could not explain this phenomenon.

The residues Asn709 and Arg806 are part of the conserved sensor 1 and sensor 2 motifs, respectively, which have been proposed to be involved in nucleotide-state sensing (Wendler et al., 2012). We therefore characterized the nucleotidebinding properties and the steady-state ATPase activity of the two sensor-motif mutants NBD2 N709A and NBD2 R806A as well as the mutant NBD2 R621Q that was used for crystallization (Fig. 4 and Table 2). Mutating the sensor 1 residue Asn709 caused only very minor changes in nucleotide binding, whereas the ATPase activity was reduced by about 30 -fold compared with the wild type. This indicates that Asn709 is of

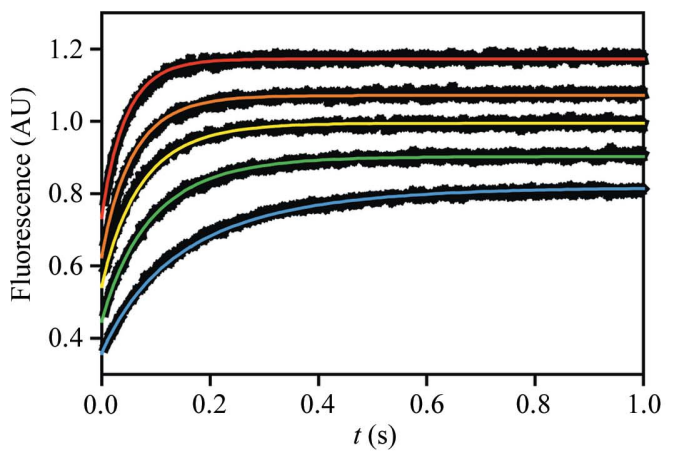

$(a)$

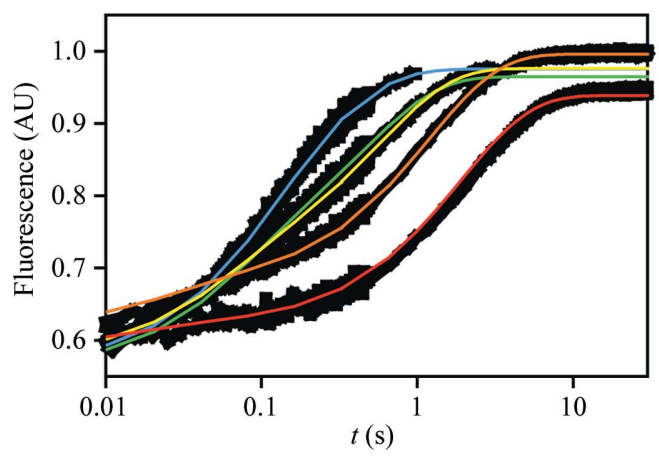

$(c)$
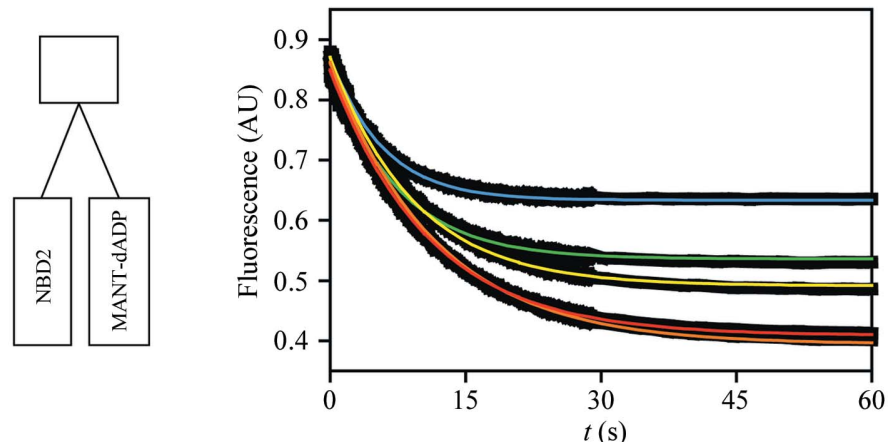

(b)

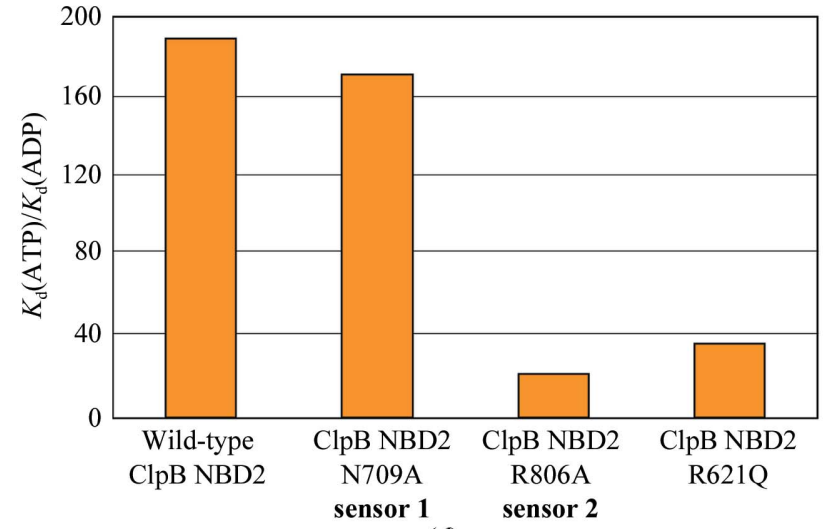

(d)

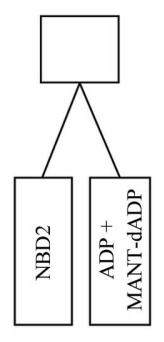

Figure 4

Nucleotide-binding kinetics measurements. The nucleotide-binding stopped-flow experiments were performed as described in $\$ 2.4$ with subsequent global data-fitting analysis according to the model given in (1). The nucleotide-binding properties of wild-type ClpB NBD2 and the N709A, R806A and R621Q mutants for MANT-dADP, MANT-dATP, ADP and ATP were determined. The kinetic traces shown exemplarily in this figure correspond to direct mixing $(a)$, displacement $(b)$ and competition $(c)$ experiments using ClpB NBD2 R806A, MANT-dADP and ADP. Coloured lines represent the fits from the global data-fitting procedure. Concentrations increase through blue, green, yellow, orange to red. The schematic experimental setup with the solutions being mixed in the different experiments is depicted next to the kinetic traces. (a) Direct mixing of $2 \mu M$ NBD2 and 5-25 $\mu M$ MANT-dADP. ( $b$ ) Displacement experiment: $2 \mu M$ ClpB NBD2 was incubated with $5 \mu M$ MANT-dADP and then mixed with 0.5-5 mM ADP. (c) ClpB NBD2 was mixed simultaneously with both MANT-dADP and ADP, giving final mixing concentrations of $0.5 \mu M$ protein, $2.5 \mu M$ MANT-dADP and 2.5-100 $\mu M$ ADP. The time axis is plotted on a logarithmic scale to account for the large range of rates in this experimental series. $(d)$ The discrimination between ADP and ATP binding is illustrated by the ratio $K_{\mathrm{d}}(\mathrm{ATP}) / K_{\mathrm{d}}(\mathrm{ADP})$. The active-site mutations R806A and R621Q significantly reduce the discrimination, primarily by impairing ADP binding. All nucleotide-binding parameters obtained from these experiments are listed in Table 2. 
Table 2

ATPase activity and nucleotide-binding parameters of ClpB NBD2 variants.

\begin{tabular}{|c|c|c|c|c|c|}
\hline \multirow[b]{2}{*}{ ClpB NBD2 variant } & \multirow{2}{*}{$\begin{array}{l}\text { ATPase } \\
\text { turnover } \\
\left(\min ^{-1}\right)\end{array}$} & \multicolumn{4}{|c|}{ Nucleotide binding } \\
\hline & & Nucleotide & $\begin{array}{l}k_{\text {on }} \\
\left(\mu M^{-1} \mathrm{~s}^{-1}\right)\end{array}$ & $k_{\text {off }}\left(\mathrm{s}^{-1}\right)$ & $K_{\mathrm{d}}(\mu M)$ \\
\hline \multirow[t]{4}{*}{ Wild-type NBD2† } & \multirow[t]{4}{*}{$8.6 \pm 2.0$} & MANT-dADP & $4.2 \pm 0.02$ & $0.015 \pm 6 \times 10^{-5}$ & $0.0035 \pm 2 \times 10^{-5}$ \\
\hline & & MANT-dATP & $3.9 \pm 0.01$ & $0.53 \pm 2 \times 10^{-3}$ & $0.14 \pm 7 \times 10^{-4}$ \\
\hline & & ADP & $2.3 \pm 0.04$ & $1.2 \pm 0.02$ & $0.51 \pm 0.01$ \\
\hline & & ATP & $0.36 \pm 0.01$ & $35 \pm 0.5$ & $97 \pm 2$ \\
\hline \multirow{4}{*}{$\begin{array}{l}\text { NBD2 N709A } \\
\text { sensor } 1 \text { mutant }\end{array}$} & \multirow{4}{*}{$0.3 \pm 0.01$} & MANT-dADP & $5.1 \pm 0.02$ & $0.012 \pm 1 \times 10^{-4}$ & $0.0024 \pm 3 \times 10^{-5}$ \\
\hline & & MANT-dATP & $4.7 \pm 0.02$ & $0.57 \pm 2 \times 10^{-3}$ & $0.12 \pm 8 \times 10^{-4}$ \\
\hline & & ADP & $2.4 \pm 0.03$ & $1.0 \pm 0.01$ & $0.43 \pm 8 \times 10^{-3}$ \\
\hline & & ATP & $0.41 \pm 0.01$ & $30 \pm 0.5$ & $74 \pm 3$ \\
\hline \multirow{4}{*}{$\begin{array}{l}\text { NBD2 R806A } \\
\text { sensor } 2 \text { mutant }\end{array}$} & \multirow{4}{*}{$0.007 \pm 0.003$} & MANT-dADP & $2.9 \pm 0.01$ & $0.080 \pm 3 \times 10^{-4}$ & $0.027 \pm 1 \times 10^{-4}$ \\
\hline & & MANT-dATP & $2.4 \pm 0.01$ & $0.71 \pm 4 \times 10^{-3}$ & $0.29 \pm 2 \times 10^{-3}$ \\
\hline & & ADP & $1.1 \pm 0.01$ & $4.4 \pm 0.05$ & $3.8 \pm 0.06$ \\
\hline & & ATP & $0.52 \pm 0.02$ & $44 \pm 1$ & $84 \pm 4$ \\
\hline \multirow[t]{4}{*}{ NBD2 R621Q } & \multirow{4}{*}{$0.3 \pm 0.04$} & MANT-dADP & $3.0 \pm 0.01$ & $0.020 \pm 2 \times 10^{-4}$ & $0.0068 \pm 8 \times 10^{-5}$ \\
\hline & & MANT-dATP & $2.7 \pm 0.01$ & $0.29 \pm 1 \times 10^{-3}$ & $0.11 \pm 7 \times 10^{-4}$ \\
\hline & & ADP & $0.94 \pm 0.01$ & $1.4 \pm 0.01$ & $1.5 \pm 0.02$ \\
\hline & & ATP & $0.40 \pm 0.01$ & $22 \pm 0.4$ & $55 \pm 2$ \\
\hline
\end{tabular}

$\dagger$ As published in Werbeck et al. (2009).

catalytic importance, but is not necessarily directly involved in nucleotide binding, which is in agreement with a previous study on the sensor 1 motif in Hsp104, the yeast homologue of ClpB (Hattendorf \& Lindquist, 2002a).

Despite being nucleotide-binding competent, the ATPase activity of the sensor 2 mutant NBD2 R806A was almost completely abolished. This is in agreement with previous studies on other AAA+ proteins, which showed that the sensor 2 arginines are catalytically essential, most likely by stabilizing intermediate states of the hydrolysis cycle (Wendler et al., 2012). Our nucleotide-binding kinetics measurements revealed a significantly reduced discrimination between ADP and ATP binding for the sensor 2 mutant NBD2 R806A, with only a very minor influence on ATP binding arising from the mutation, but significantly impaired ADP binding (Fig. 4d). This result hints towards a direct interaction of Arg806 with the nucleotide that contributes to the binding energy in the ADP state but not in the ATP state, which is discussed in further detail in the following section with respect to the structural data available. Previous results from equilibrium titrations with Hsp104 by Lindquist and coworkers suggested similarly impaired nucleotide binding for both ADP and ATP in the presence of the sensor 2 mutation R826M in the oligomeric context of the full-length protein (Hattendorf \& Lindquist, 2002b). Our approach using only the isolated nucleotide-binding domain 2 (NBD2) of ClpB allows a more detailed kinetic analysis of the nucleotide-binding behaviour that is able to capture the differences in nucleotide discrimination.

The mutant variant NBD2 R621Q is nucleotide-binding competent, but shows an approximately 30-fold reduced ATPase activity. It is highly likely that Arg621 is involved in stabilizing negative charges, especially in the transition state. The R621Q mutation significantly reduces the discrimination between ATP and ADP, mainly by impaired ADP binding, but also owing to a slight increase in ATP binding (Fig. $4 d$ and Table 2). As discussed before for NBD2 R806A, it is possible that Arg621 functions as a nucleotide sensor, adopting alternative conformations dependent on the nucleotide-binding state. Arg621 is highly conserved in ClpB and Hsp104 homologues from different organisms, as well as related $\mathrm{AAA}+$ proteins that possess a second C-terminal nucleotidebinding domain, such as ClpA and ClpC. A potential mechanistic role of Arg621 is discussed further in the next section, which introduces the high-resolution X-ray structure of ClpB NBD2 R621Q in complex with the nonhydrolysable ATP analogue AMPPCP.

\subsection{Mechanistic insights from the structure of ClpB NBD2 R621Q bound to an ATP analogue with the essential $\mathrm{Mg}^{2+}$ ion incorporated into the catalytic site}

3.4.1. The role of Arg621 in $\mathbf{M g}^{2+}$ incorporation. Analysis of the ClpB NBD2 K601Q structure (see Fig. 3b) led to the hypothesis that the highly conserved residue Arg621 might interfere with the binding of $\mathrm{Mg}^{2+}$ to the phosphates of the bound nucleotide in the catalytic site. In at least one of its possible conformations, the Arg621 side chain positions a positive charge close to the expected $\mathrm{Mg}^{2+}$-binding site and thereby competes with $\mathrm{Mg}^{2+}$ incorporation. The mutation R621Q was therefore introduced to facilitate the incorporation of the essential $\mathrm{Mg}^{2+}$ into the $\mathrm{ClpB}$ NBD2 structure. Crystallization of ClpB NBD2 R621Q in the presence of the nonhydrolysable ATP analogue AMPPCP and $\mathrm{MgCl}_{2}$ yielded well diffracting diamond-shaped crystals in space group $\mathrm{Pb}_{5}$ (Table 1). The structure was determined to a resolution of $1.7 \AA$ and for the first time we could unambiguously locate the essential $\mathrm{Mg}^{2+}$ ion between the $\beta$ - and $\gamma$-phosphates of the bound AMPPCP (Fig. $5 a$ ). The $\mathrm{Mg}^{2+}$ ion is coordinated octahedrally by the two phosphate groups, the hydroxyl group of the Walker A residue Thr602 and three water molecules. The Walker B residue Asp667 forms hydrogen bonds to two of the hydration-shell water molecules. Additionally, we observe electron density for a second $\mathrm{Mg}^{2+}$ ion coordinated octahed- 
rally by six water molecules at a distance of $4.5 \AA$ to the $\gamma$-phosphate of the bound AMPPCP. We assume that this additional $\mathrm{Mg}^{2+}$ ion is not of mechanistic relevance but rather is incorporated owing to the R621Q mutation and the nonphysiologically high $\mathrm{MgCl}_{2}$ concentration of $75 \mathrm{mM}$ used in the crystallization cocktail. Indeed, when comparing the Arg621 side-chain conformations in the different structures available, two alternative positions are observed. The positively charged side-chain guanidinium group is either located close to the nucleotide, where it blocks the incorporation of the essential $\mathrm{Mg}^{2+}$ ion, or close to where the second (most likely nonphysiological) $\mathrm{Mg}^{2+}$ ion is found in the NBD2 R621Q structure (Fig. 5c). The negatively charged environment that surrounds this second position (formed by Asp623, Asp667 and Glu668) can easily stabilize a positive charge, be it the Arg621 side chain in the wild-type protein or the second $\mathrm{Mg}^{2+}$ ion incorporated into this position owing to the R621Q mutation. To verify this hypothesis, we repeated the crystallization experiment in the presence of only $10 \mathrm{mM} \mathrm{MgCl}_{2}$ and additionally soaked with $100 \mathrm{~m} M$ guanidinium chloride ( $\mathrm{GdmCl})$, which was supposed to function as a mobile substitute for the arginine side chain that was removed owing to the R621Q mutation. Indeed, in the structure obtained from crystals grown in this condition, only one $\mathrm{Mg}^{2+}$ ion is present, which occupies the expected position between the $\beta$ - and $\gamma$-phosphates of the bound AMPPCP. Additionally, a $\mathrm{Gdm}^{+}$ ion is localized where the second $\mathrm{Mg}^{2+}$ ion was bound before, suggesting that this position would usually be occupied by the Arg621 side chain (Fig. 5b).
Based on this structural analysis and the kinetic data showing a reduced discrimination between ADP and ATP binding in ClpB NBD2 R621Q (Fig. $4 d$ and Table 2), we propose that Arg621 is involved in nucleotide sensing and the incorporation of the essential $\mathrm{Mg}^{2+}$ ion into the catalytic site which is crucial for efficient ATP hydrolysis. Arg621 adopts two different conformations, which are presumably dependent on the stage of the ATPase cycle (Fig. 5c). Since the R621Q mutation selectively impairs ADP binding, we conclude that in the ADP state Arg621 contributes significantly to the nucleotide-binding energy, presumably by mimicking the nucleotide-associated $\mathrm{Mg}^{2+}$. In the ATP state, Arg621 is most likely to adopt the position further away from the nucleotide, thereby not contributing to the binding energy but allowing the incorporation of the essential $\mathrm{Mg}^{2+}$ ion. Regulatory metal switches have also been found in other ATPases, such as the DNA mismatch-repair protein MutS, in which the essential Walker B motif is rearranged in the absence of $\mathrm{Mg}^{2+}$ (Lebbink et al., 2010), which is reminiscent of the situation that we observe for ClpB NBD2. We therefore hypothesize that a variation of a metal-switch mechanism with Arg621 as a key regulatory feature is implemented in ClpB NBD2.

3.4.2. Nucleotide state-sensing mechanisms. The sensor 1 and 2 motif residues Asn709 and Arg806, respectively, are catalytically essential and have been proposed to be involved in sensing and communicating different nucleotide states (Guenther et al., 1997). The structural basis of how this function is fulfilled, however, is poorly understood. Owing to the high resolution of $1.7 \AA$, we were able to assign alternative

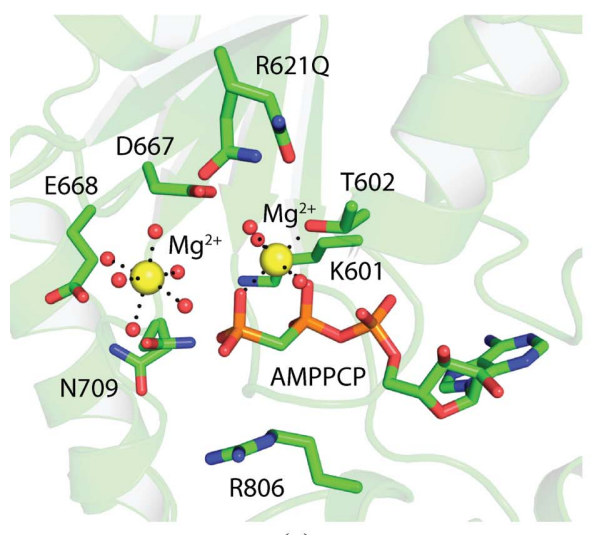

(a)

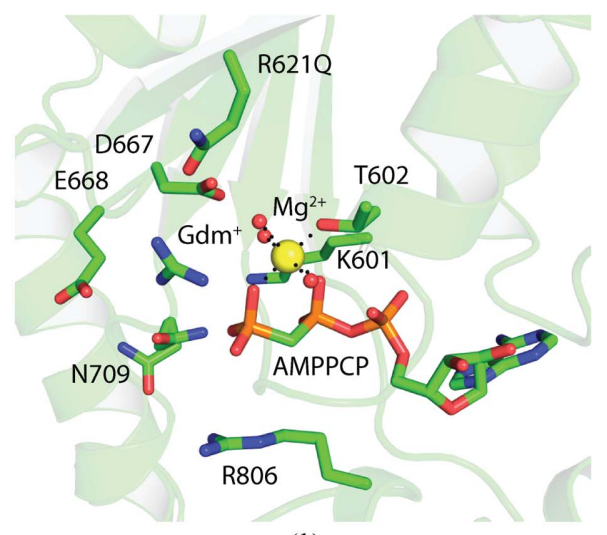

(b)

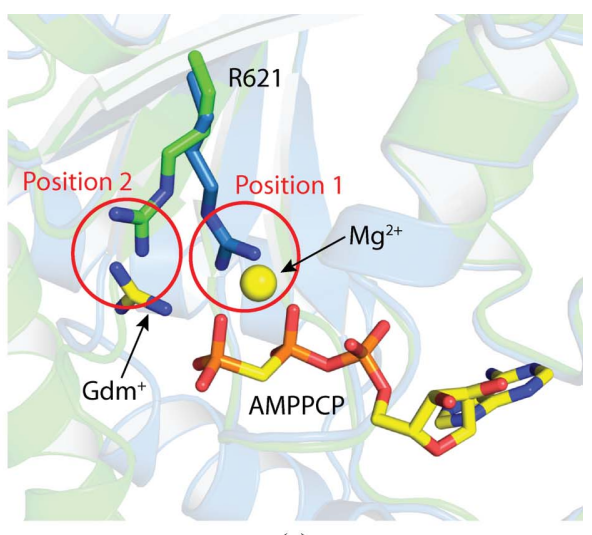

(c)

Figure 5

The active-site mutation R621Q facilitates the incorporation of $\mathrm{Mg}^{2+}$ into ClpB NBD2. (a) Structure of ClpB NBD2 R621Q in complex with AMPPCP. The essential $\mathrm{Mg}^{2+}$ ion is coordinated by the $\beta$ - and $\gamma$-phosphates of the bound nucleotide, the Walker A threonine Thr602 and three water molecules. Owing to the mutation and the high $\mathrm{MgCl}_{2}$ concentration of $75 \mathrm{mM}$ in the crystallization solution, a second $\mathrm{Mg}^{2+}$ ion with a complete hydration shell $[d(\mathrm{Mg}-\mathrm{O})=2.0-2.3 \AA]$ is present in the catalytic site at a distance of $4.5 \AA$ from the $\gamma$-phosphate. The Walker A lysine Lys601 adopts the stretched conformation observed in the previous structures and does not contact the nucleotide. The Walker B aspartate Asp667 forms hydrogen bonds to water molecules of the hydration shell of the essential $\mathrm{Mg}^{2+}$ ion. However, there is no significant rearrangement of the catalytic site compared with the $\mathrm{Mg}$-free structures. Residues 621 and 709 were modelled using alternative conformations, which are both shown in the figure. (b) Structure of ClpB NBD2 R621Q in complex with AMPPCP and $\mathrm{GdmCl}$. Using a lower $\mathrm{MgCl}_{2}$ concentration of $10 \mathrm{~m} M$ and additionally soaking with $100 \mathrm{~m} M \mathrm{GdmCl}$, the second $\mathrm{Mg}^{2+}$ ion was replaced by a $\mathrm{Gdm}^{+}$ion, whereas the essential $\mathrm{Mg}^{2+}$ ion bound at the phosphates of the nucleotide remained. The $\mathrm{Gdm}^{+}$ion represents a mobile arginine side-chain mimic, suggesting that the mutated Arg621 would usually occupy this position. Residue 709 was modelled using alternative conformations, which are both shown in the figure. (c) Superposition of the two crystal forms observed for ClpB NBD2 showing two different positions for the conserved active-site residue Arg621 (green, wild-type NBD2 + AMPPCP; blue, NBD2 K601Q + MANT-dADP). Additionally, the AMPPCP, the essential $\mathrm{Mg}^{2+}$ ion and the $\mathrm{Gdm}^{+}$ion of the NBD2 R621Q structure shown in $(b)$ are overlaid in yellow, illustrating the two possible positions of the Arg621 side chain. In position 1, Arg621 is located close to the nucleotide and impairs $\mathrm{Mg}^{2+}$ incorporation. In position 2, it is at a greater distance from the nucleotide, allowing $\mathrm{Mg}^{2+}$ binding. 
conformations for certain residues in the structure of $\mathrm{ClpB}$ NBD2 R621Q in complex with AMPPCP. We identified a hydrogen-bonding network between sensor 1 residue Asn709, P-loop residue Thr597 and the $\gamma$-phosphate of the bound nucleotide. Depending on its orientation, Thr597 can either form a hydrogen bond to the nucleotide or to the side chain of Asn709 (Fig. 6a). One can speculate that in the ATP state

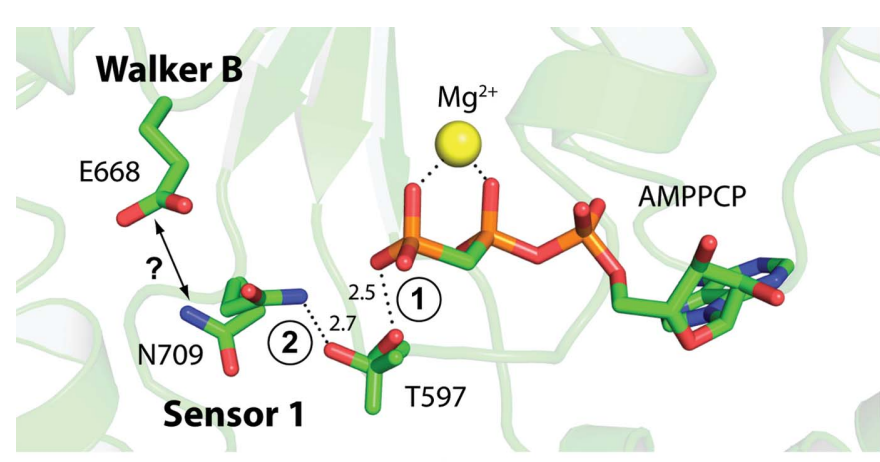

(a)
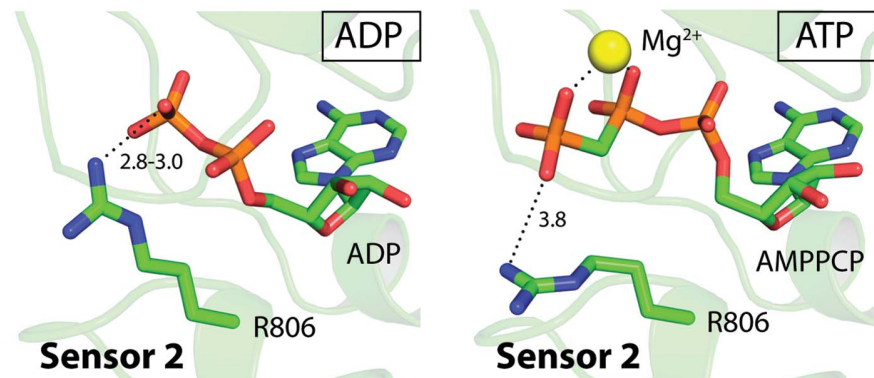

Figure 6

(b)

Nucleotide-state sensing in ClpB NBD2. (a) Sensor 1 motif: structure of ClpB NBD2 R621Q in complex with AMPPCP showing alternative conformations for the P-loop residue Thr597 and the sensor 1 residue Asn709, which are involved in a hydrogen-bonding network around the nucleotide. Depending on the nucleotide state, Thr597 can form a hydrogen bond to either the $\gamma$-phosphate of ATP (1) or, in the absence of the $\gamma$-phosphate, to sensor 1 residue Asn709 (2). Currently, it is unclear how such a switch is further communicated through the molecule. However, it has been proposed previously that the sensor 1 motif is involved in hydrolysis, coordinating the attacking water together with the Walker B glutamate Glu668 (Gai et al., 2004). This might only be possible when Asn709 is not captured in the interaction with Thr597; thus, in the ATP-bound state. Distances are given in $\AA$. (b) Sensor 2 motif: ClpB NBD2 in the ADP and ATP state, respectively, showing different positions of the sensor 2 residue Arg806, which is not influenced by crystal contacts in the structures shown in this figure. In the ADP state (left panel; ClpB NBD2 R621Q + ADP), Arg806 interacts with the $\beta$-phosphate of the nucleotide $(d=3.0 \AA)$, which is also the case in the MANT-dADP-bound structure $(d=2.8 \AA)$ shown in Fig. $3(b)$ and in another recently published ADP-bound NBD2 structure $(d=2.8 \AA$; Biter et al., 2012). This direct interaction between Arg806 and the nucleotide in the ADP state contributes to the nucleotide-binding energy, which is in agreement with the observation that the R806A mutation causes impaired ADP binding (Table 2). In the ATP state (right panel; ClpB NBD2 R621Q + AMPPCP + GdmCl), Arg806 must change its position because it would otherwise clash with the $\gamma$-phosphate. Indeed, it bends away from the phosphates $(d=3.8 \AA$ ) , thereby no longer contributing to the nucleotide binding energy significantly, which is supported by our nucleotide-binding kinetics measurements showing that the R806A mutation has no significant effect on ATP binding (Table 2). The clearly different behaviour of Arg806 in the presence of ADP compared with ATP demonstrates that this residue functions as a bona fide nucleotide sensor.
Thr597 preferentially interacts with the $\gamma$-phosphate. In the nucleotide-free or ADP state, when no $\gamma$-phosphate is present, it rotates and interacts with Asn709. This putative switch might be of regulatory importance. Even though the sensor 1 mutation N709A causes no significant changes in the nucleotide-binding properties, it severely impairs ATP hydrolysis. This is in agreement with the hypothesis that the sensor 1 residue works in concert with the Walker B motif in activating the attacking water molecule during hydrolysis (Gai et al., 2004). A nucleotide-dependent conformational switch of Asn709 might be the regulating element for this function. However, the structural data available are not sufficient to unambiguously confirm this hypothesis. Based on the kinetic data, we can state that the essential sensor 1 motif (Asn709) is not directly involved in nucleotide binding, which is inconsistent with the role of a classic nucleotide sensor. This result is in agreement with a previous study by Hattendorf $\&$ Lindquist (2002a).

In contrast, our characterization of the sensor 2 motif (Arg806) provides clear evidence for direct nucleotide sensing and discrimination. In addition to the AMPPCP-bound structure of ClpB NBD2 R621Q in the presence of $\mathrm{MgCl}_{2}$ and $\mathrm{GdmCl}$, we also obtained the structure of this mutant variant in the ADP-bound state to a resolution of $2.1 \AA$ (Table 1). Since the sensor 2 residue Arg806 was no longer trapped in a crystal contact in these structures, we could identify two different conformations of Arg806 that are adopted depending on the nucleotide state (Fig. $6 b$ ). In the ADP state, the side chain of Arg806 interacts directly with the $\beta$-phosphate of the bound nucleotide $(d<3.0 \AA)$, which is also observed in both our MANT-dADP-bound structure and the recently published NBD2 structure (PDB entry 4fcw; Biter et al., 2012). In the ATP state, the Arg806 side chain must adopt an alternative conformation because otherwise it would clash with the $\gamma$-phosphate. Indeed, Arg806 is located about $4 \AA$ away from the nucleotide in the AMPPCP-bound structure. This is in agreement with our nucleotide-binding data, which show that the sensor 2 mutation R806A causes significantly impaired ADP binding but almost no change in ATP binding. It can be concluded that the direct interaction with the nucleotide in the ADP state contributes to the binding energy, whereas in the ATP state no interaction between Arg806 and the nucleotide takes place. This, together with the function of residue Arg621 described in the previous sections, provides a veritable explanation for the observed discrimination between ADP and ATP binding in ClpB NBD2. The combination of structural and kinetic analysis further defines the role of the sensor 2 residue Arg806 in nucleotide-state sensing and identifies one clear difference between the ADP and ATP state, which is crucial for understanding the mode of action of this ATPase motor domain.

\subsection{Comparison with other AAA+ protein structures regarding the hexameric context}

To evaluate the conclusions drawn from the ClpB NBD2 structures presented in this work, it is crucial to discuss the 
results in the light of the oligomeric context of the holoprotein. Even though smaller oligomeric species, as present for the separate NBD2 construct, hydrolyze ATP efficiently, full disaggregation activity is most likely to require a hexameric assembly. There are several structural studies available that show hexameric states of AAA+ proteins, for example for

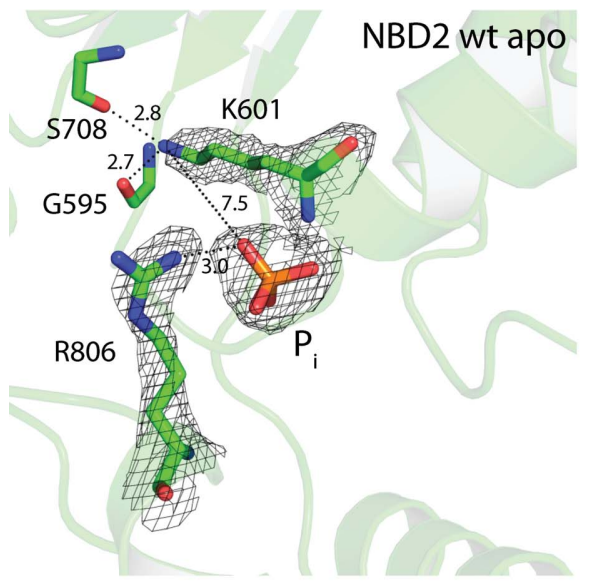

$(a)$

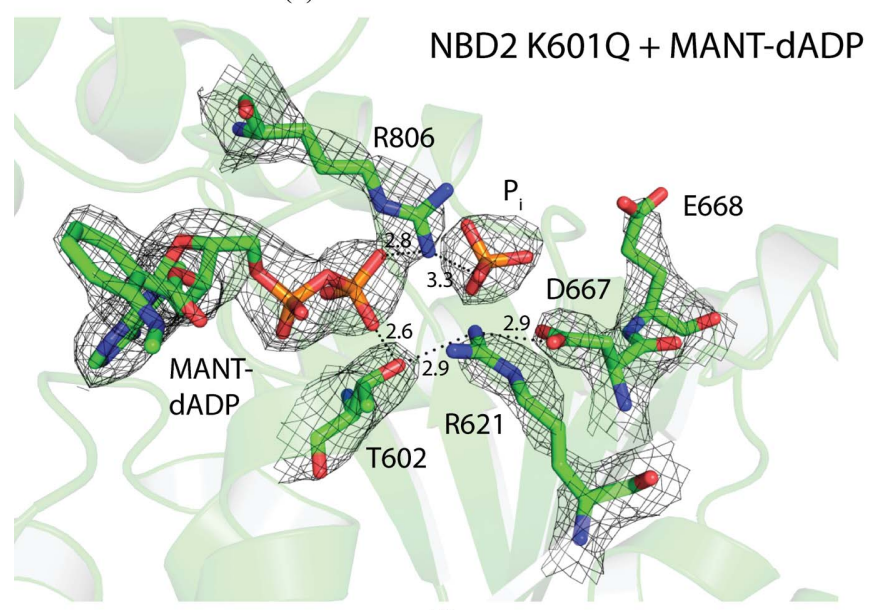

(d)

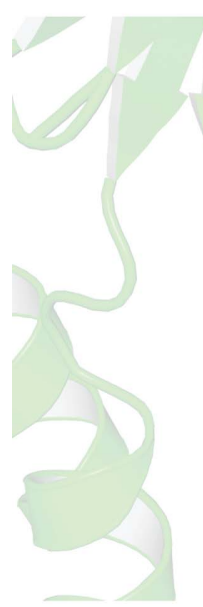

Figure 7

Overview of the states captured for ClpB NBD2. $2 m F_{\mathrm{o}}-D F_{\mathrm{c}}$ electron density (contour levels are given below) is shown for relevant parts of each structure, such as the bound nucleotide and conserved Walker or sensor residues. $\mathrm{Mg}^{2+}$ ions are shown in yellow, including their octahedral coordination sphere with $d(\mathrm{Mg}-\mathrm{O})=2.0-2.3 \AA$. Water molecules are shown as red spheres. Distances are given in $\AA$. (a) Wild-type ClpB NBD2, nucleotide-free, PDB entry 4lj4, contour level 1.1 $\sigma$. (b) Wild-type ClpB NBD2 in complex with ADP, PDB entry 4lj5, contour level 1.5 $\sigma$. (c) Wild-type ClpB NBD2 in complex with AMPPCP, PDB entry 4lj6, contour level 1.5 $\sigma .(d)$ ClpB NBD2 K601Q in complex with MANT-dADP, PDB entry 4lj7, contour level 1.1 $\sigma .(e)$ ClpB NBD2 R621Q in complex with AMPPCP, PDB entry 4lj9, contour level 1.5 $\sigma$. $(f)$ ClpB NBD2 R621Q in complex with ADP, PDB entry 4lj8, contour level 1.5 $\sigma .(g)$ ClpB NBD2 R621Q in complex with AMPPCP and GdmCl, PDB entry 4lja, contour level 1.5 $\sigma$. For detailed structure analyses, compare Figs. 2, 3,5 and 6.

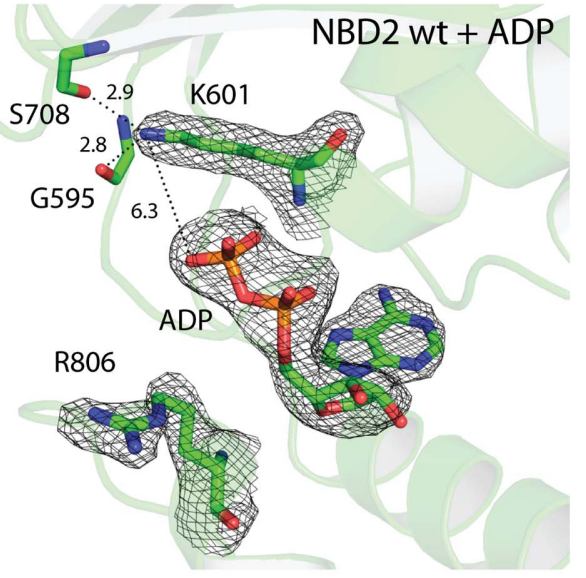

$(b)$

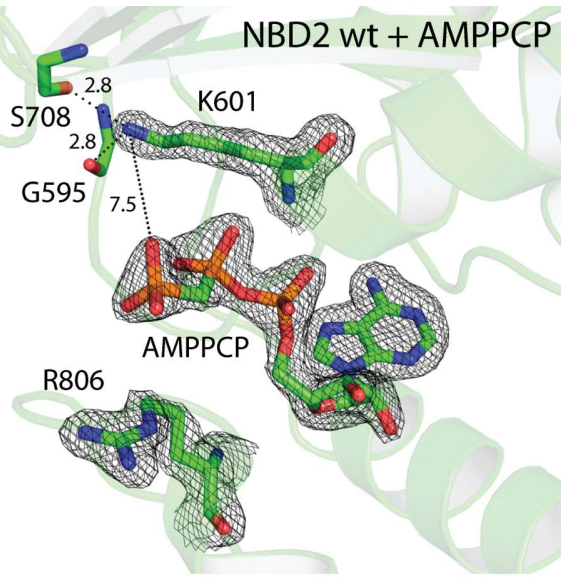

(c)

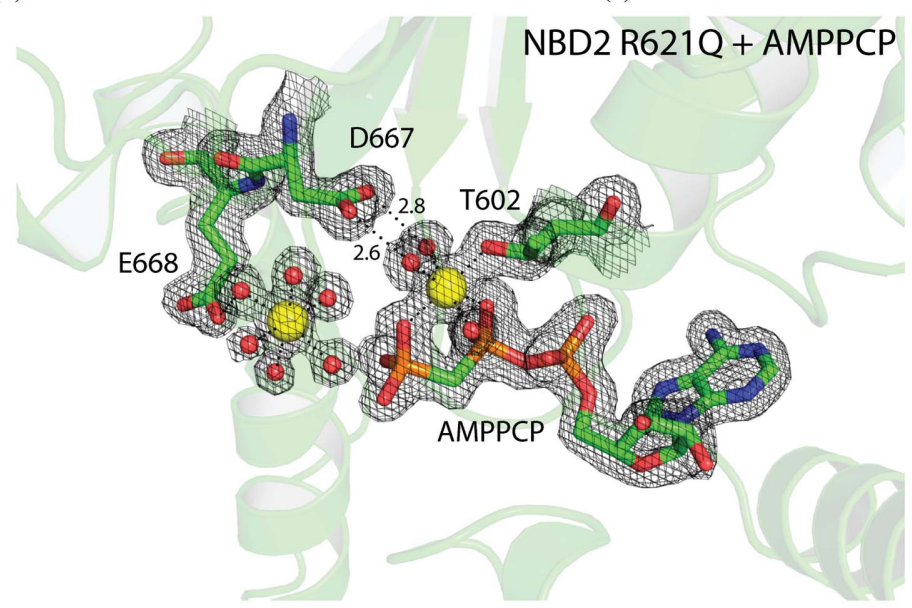

(e)

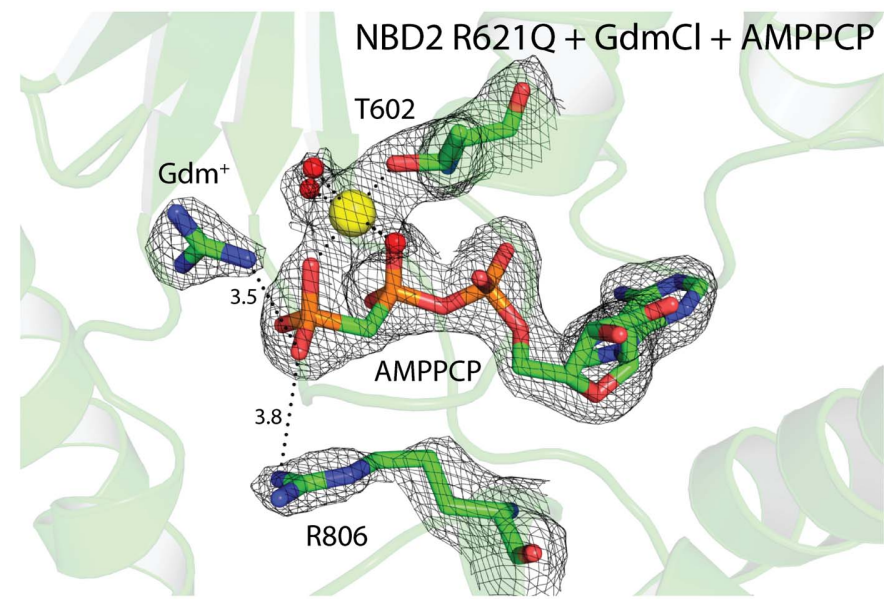

$(g)$ 
p97, ClpX, SV40 LTag and ClpC. Despite a common overall architecture, the essential interactions and resulting structural changes in the oligomeric arrangement upon ATP hydrolysis vary remarkably for the different AAA+ proteins. For $\mathrm{ClpC}$, which shares high sequence identity with $\mathrm{ClpB}$, a planar hexameric state in complex with the adaptor protein MecA has been observed in both X-ray structures as well as cryo-EM studies (Liu et al., 2013; Wang et al., 2011). Unfortunately, the resolution is not sufficient to discuss detailed side-chain interactions. For the AAA+ helicase LTag from SV40, structures of the planar hexamer are available for different nucleotide states at very high resolution (up to $1.9 \AA$ Å), which allowed a detailed mechanistic analysis (Gai et al., 2004). Here, a conserved lysine from the neighbouring subunit works in concert with the classic arginine finger. Furthermore, extensive mechanistic and structural studies regarding the hexameric assembly have been performed for ClpX, another prominent AAA+ unfoldase (Glynn et al., 2009, 2012; Martin et al., 2005; Stinson et al., 2013), and for p97, an ERADassociated AAA+ protein (Davies et al., 2008; Li et al., 2012; Nishikori et al., 2011).

To evaluate our results, we compared the average distances between the nucleotide and the catalytically relevant Walker and sensor residues in our structures with those observed in the available high-resolution hexameric AAA+ structures, using the analysis presented by Wendler and coworkers as a starting point (Wendler et al., 2012). For instance, the distance between the side-chain carbonyl $\mathrm{O}$ atom of the Walker $\mathrm{B}$ aspartate and the $\gamma$-phosphate $\mathrm{O}$ atom of the nucleotide ranges from 3.0 to $5.5 \AA$ in published hexameric structures and is $4.5 \AA$ in the case of ClpB NBD2 R621Q + AMPPCP. Similarly, the distance between the nucleotide and the sensor 1 asparagine side chain ranges from $3.1 \AA$ for SV40 LTag (PDB entry 1svm; Gai et al., 2004) to $6.0 \AA$ for p97 (PBD entry 3cf1; Davies et al., 2008), and is $4.6 \AA$ in the case of ClpB NBD2. Sensor 2 motifs are only present in a subset of AAA+ proteins. We compared ClpB NBD2 with ClpX (PDB entry 3hws; Glynn et al., 2009) and also found similar distances and orientations regarding the sensor 2 arginine. Even though we cannot exclude that our single NBD2 structures somewhat differ from their counterparts in the hexamer, this comparison indicates that they may very well represent snapshots of relevant states that are also adopted in the hexameric context.

It is remarkable that all of the structures of ClpB (fulllength and NBD2) available to date show a helical arrangement of subunits representing a spiral-like oligomeric state, which is also observed in the available high-resolution structure of ClpA (Guo et al., 2002), a closely related unfoldase that also bears two NBDs per molecule. Even though cryo-EM data suggest that the planar hexameric ring is the predominant active form, it cannot be excluded that spirals are present in solution and are of certain mechanistic relevance. A possible biological role of a spiral has actually been discussed for other AAA+ proteins (Erzberger \& Berger, 2006).

Even though the exact arrangement of the functionally relevant oligomeric state in $\mathrm{ClpB}$ remains elusive, our NBD2 structures contribute to the overall mechanistic understanding by providing structural details at high resolution for one separate motor building block of an AAA+ machine in different states. A summary of the different states captured for ClpB NBD2 is presented in Fig. 7.

\subsection{Various P-loop proteins exhibit similar inactive conformations: a potential regulatory function of the Walker A lysine as a molecular switch}

Even though we were finally able to incorporate the essential $\mathrm{Mg}^{2+}$ ion into the structure of ClpB NBD2, the

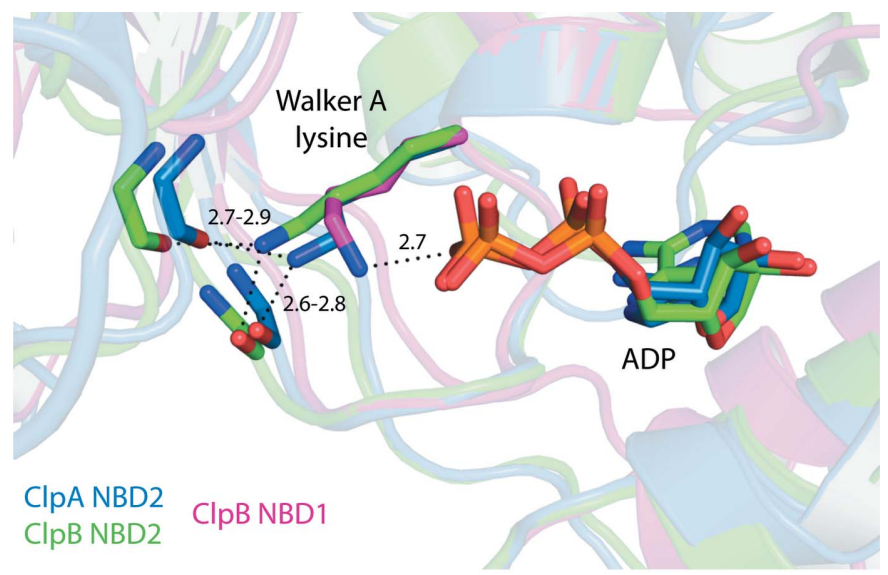

(a)

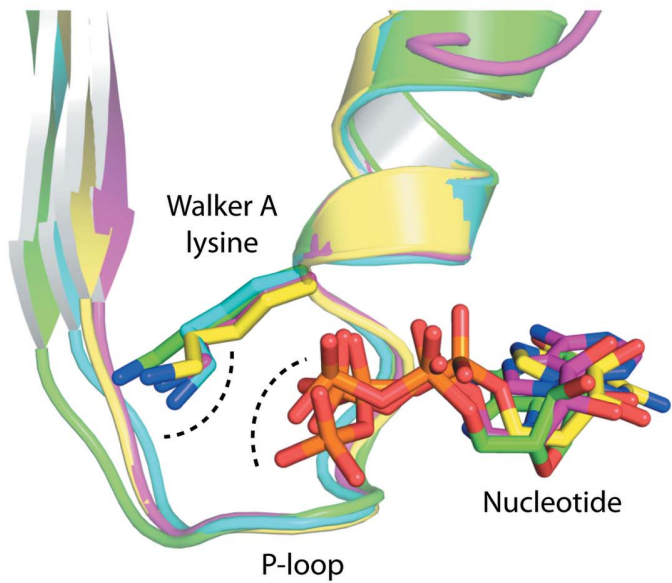

(b)

Figure 8

Various P-loop protein structures exhibit an inactive catalytic site conformation. (a) P-loop superposition of ClpA NBD2 (blue, PDB entry 1r6b; Xia et al., 2004) and ClpB NBD2 (green) in complex with ADP. The closely related AAA+ protein ClpA shows an almost identical catalytic site conformation as $\mathrm{ClpB}$, characterized by a stretched Walker A lysine in NBD2 that contacts carbonyl backbone groups. Notably, the corresponding Walker A lysine in NBD1 of both proteins directly interacts with the nucleotide in the conventional manner, which is shown for ClpB NBD1 (pink, PDB entry 4hse; Zeymer et al., 2013). (b) P-loop superposition of nucleotide-bound structures of different P-loop proteins: ClpB NBD2 (green), the kinesin family member Kin10 (pink; PDB entry 3pxn; Cochran et al., 2011), the human GTPase GIMAP2 that controls apoptosis in lymphocytes (yellow; PDB entry 2xtn; Schwefel et al., 2010) and the DNA mismatch-repair protein MutS (blue; PDB entry 3k0s; Lebbink et al., 2010). The Walker A lysine residues adopt a stretched conformation and interact with carbonyl backbone groups but not with the nucleotide, thereby stabilizing an open inactive conformation of the catalytic site. 
overall conformation of the catalytic site remained open and therefore inactive. The Walker A lysine Lys601 is still stretched and contacts P-loop backbone carbonyl groups but not the nucleotide. We therefore analyzed the structures of other P-loop proteins available at sufficiently high resolutions and found more examples of such open inactive catalytic site conformations characterized by a stretched Walker A lysine. In the structure of a closely related AAA+ protein, ClpA, the situation is almost identical (PDB entry 1r6b; Xia et al., 2004). Whereas in NBD1 of both of the proteins ClpA and ClpB the Walker A lysine directly interacts with the nucleotide in the conventional way, in NBD2 the stretched Walker A lysine conformation is observed (Fig. 8a). However, more distant relatives in the large family of P-loop proteins were also found to adopt this open inactive conformation of the catalytic site in their respective crystal structures. Three examples are shown in Fig. 8(b): the kinesin family member Kin10 in complex with ADP (PDB entry 3pxn; Cochran et al., 2011), a human GTPase controlling apoptosis in lymphocytes, GIMAP2, in complex with GTP (PDB entry 2xtn; Schwefel et al., 2010) and the DNA mismatch-repair protein MutS in complex with ADP (PDB entry 3k0s; Lebbink et al., 2010). Interestingly, in the latter example the inactive catalytic site arrangement is also coupled to the absence of $\mathrm{Mg}^{2+}$. We hypothesize that the open catalytic site conformations represent an inactive resting state during the ATPase cycle, which is often captured in X-ray structures and may be of regulatory importance. Zhang and Wigley proposed previously that a so-called 'glutamate switch' directly controls the conformation of the essential Walker B glutamate residue in AAA+ domains, keeping it in an inactive state by hydrogen bonding to a polar residue located on the tip of $\beta$-strand $\beta 2$ (Zhang \& Wigley, 2008). In ClpB NBD2 from T. thermophilus, this residue corresponds to a threonine (Thr625). However, we do not observe a direct hydrogenbonding interaction with the Walker B glutamate Glu668 in our structures. Instead, we suggest a different mechanism that forces the catalytically essential residues, including the Walker B glutamate, into an inactive position. We propose that the Walker A lysine in its stretched conformation acts like a 'molecular doorstop' that keeps the catalytic site open and thereby inactive in order to avoid unnecessary ATP turnover, for example in the absence of a secondary substrate. Many ATPases have a low basal ATP-hydrolysis activity which is significantly stimulated upon addition of substrate, with the highly conserved Walker A lysine having a catalytic role. We propose a second, regulatory function for the Walker A lysine, where it is involved in switching between an inactivated, 'fuelsaving' state and the conventional active state of a molecular motor.

The question arises as to why this regulatory mechanism is only implemented in a subset of AAA+ domains. In ClpB, the stretched Walker A lysine conformation is observed exclusively in the C-terminal nucleotide-binding domain NBD2, but not in the N-terminal NBD1. In the current mechanistic view, NBD2 is the actual motor of the ClpB disaggregation machine, whereas NBD1 is mainly of regulatory importance, interacting with the co-chaperone DnaK via the unique coiled-coil middle domain, which turns out to be a highly sophisticated regulatory element of ClpB (Rosenzweig et al., 2013; Oguchi et al., 2012). Thus, NBD1 is already highly regulated, whereas the strong motor NBD2 needs efficient regulation, which we propose is provided by a conformational switch of the Walker A lysine that is likely to be induced by binding of the substrate protein.

\section{Conclusions}

Our structure-based analysis of ClpB NBD2, a representative of the AAA+ motor domains, reveals mechanistic insights into nucleotide sensing and the regulation of the ATPase activity. We show how the conserved sensor 2 motif directly contributes to the discrimination between ADP and ATP binding. Furthermore, we suggest that a conserved active-site arginine regulates the incorporation of the essential $\mathrm{Mg}^{2+}$ ion and is therefore essential for the functioning of the ATPase cycle. In addition, we propose a regulatory switch where the conserved Walker A lysine, when present in a fully stretched conformation, causes an inactive arrangement of the catalytic site to avoid unnecessary ATPase turnover.

We thank Sabine Zimmermann and Jessica Eschenbach for excellent technical assistance and Anton Meinhart for help with in-house X-ray data collection. We are indebted to Anna Scherer, Victor Kulik and Philipp Beinker for contributions during the initial stages of the project. We are grateful to the $\mathrm{X}$-ray data-collection teams from the Max Planck Institutes in Heidelberg and Dortmund, who performed the measurements at the Swiss Light Source (SLS). We also thank the SLS staff for their support in setting up the beamline and Ingrid Vetter and Chris Roome for excellent support with the crystallographic software. The project was funded by the Max Planck Society and a PhD scholarship to $\mathrm{CZ}$ from the German National Academic Foundation.

\section{References}

Beinker, P., Schlee, S., Auvula, R. \& Reinstein, J. (2005). J. Biol. Chem. 280, 37965-37973.

Biter, A. B., Lee, S., Sung, N. \& Tsai, F. T. (2012). Proc. Natl Acad. Sci. USA, 109, 12515-12520.

Brünger, A. T. (1990). Acta Cryst. A46, 46-57.

Chen, V. B., Arendall, W. B., Headd, J. J., Keedy, D. A., Immormino, R. M., Kapral, G. J., Murray, L. W., Richardson, J. S. \& Richardson, D. C. (2010). Acta Cryst. D66, 12-21.

Cochran, J. C., Zhao, Y. C., Wilcox, D. E. \& Kull, F. J. (2011). Nature Struct. Mol. Biol. 19, 122-127.

Davies, J. M., Brunger, A. T. \& Weis, W. I. (2008). Structure, 16, 715-726.

Doyle, S. M. \& Wickner, S. (2009). Trends Biochem. Sci. 34, 40-48.

Emsley, P. \& Cowtan, K. (2004). Acta Cryst. D60, 2126-2132.

Engh, R. A. \& Huber, R. (1991). Acta Cryst. A47, 392-400.

Erzberger, J. P. \& Berger, J. M. (2006). Annu. Rev. Biophys. Biomol. Struct. 35, 93-114.

Franzmann, T. M., Czekalla, A. \& Walter, S. G. (2011). J. Biol. Chem. 286, 17992-18001.

Gai, D., Zhao, R., Li, D., Finkielstein, C. V. \& Chen, X. S. (2004). Cell, 119, 47-60.

Glynn, S. E., Martin, A., Nager, A. R., Baker, T. A. \& Sauer, R. T. (2009). Cell, 139, 744-756. 
Glynn, S. E., Nager, A. R., Baker, T. A. \& Sauer, R. T. (2012). Nature Struct. Mol. Biol. 19, 616-622.

Guenther, B., Onrust, R., Sali, A., O’Donnell, M. \& Kuriyan, J. (1997). Cell, 91, 335-345.

Guo, F., Maurizi, M. R., Esser, L. \& Xia, D. (2002). J. Biol. Chem. 277, 46743-46752.

Hanson, P. I. \& Whiteheart, S. W. (2005). Nature Rev. Mol. Cell Biol. 6, 519-529.

Hattendorf, D. A. \& Lindquist, S. L. (2002a). EMBO J. 21, 12-21.

Hattendorf, D. A. \& Lindquist, S. L. (2002b). Proc. Natl Acad. Sci. USA, 99, 2732-2737.

Hayward, S. \& Lee, R. A. (2002). J. Mol. Graph. Model. 21, 181-183.

Kabsch, W. (2010). Acta Cryst. D66, 125-132.

Kuzmic, P. (1996). Anal. Biochem. 237, 260-273.

Lebbink, J. H., Fish, A., Reumer, A., Natrajan, G., Winterwerp, H. H. \& Sixma, T. K. (2010). J. Biol. Chem. 285, 13131-13141.

Lee, S., Choi, J.-M. \& Tsai, F. T. (2007). Mol. Cell, 25, 261-271.

Lee, S., Sowa, M. E., Watanabe, Y.-H., Sigler, P. B., Chiu, W., Yoshida, M. \& Tsai, F. T. F. (2003). Cell, 115, 229-240.

Li, G., Huang, C., Zhao, G. \& Lennarz, W. J. (2012). Proc. Natl Acad. Sci. USA, 109, 3737-3741.

Liu, J., Mei, Z., Li, N., Qi, Y., Xu, Y., Shi, Y., Wang, F., Lei, J. \& Gao, N. (2013). J. Biol. Chem. 288, 17597-17608.

Martin, A., Baker, T. A. \& Sauer, R. T. (2005). Nature (London), 437, 1115-1120.

McCoy, A. J., Grosse-Kunstleve, R. W., Adams, P. D., Winn, M. D., Storoni, L. C. \& Read, R. J. (2007). J. Appl. Cryst. 40, 658-674.

McRee, D. E. (1999). J. Struct. Biol. 125, 156-165.

Murshudov, G. N., Skubák, P., Lebedev, A. A., Pannu, N. S., Steiner, R. A., Nicholls, R. A., Winn, M. D., Long, F. \& Vagin, A. A. (2011). Acta Cryst. D67, 355-367.

Nishikori, S., Esaki, M., Yamanaka, K., Sugimoto, S. \& Ogura, T. (2011). J. Biol. Chem. 286, 15815-15820.

Oguchi, Y., Kummer, E., Seyffer, F., Berynskyy, M., Anstett, B., Zahn, R., Wade, R. C., Mogk, A. \& Bukau, B. (2012). Nature Struct. Mol. Biol. 19, 1338-1346.

Ogura, T., Whiteheart, S. W. \& Wilkinson, A. J. (2004). J. Struct. Biol. 146, 106-112.

Rosenzweig, R., Moradi, S., Zarrine-Afsar, A., Glover, J. R. \& Kay, L. E. (2013). Science, 339, 1080-1083.
Sanchez, Y. \& Lindquist, S. L. (1990). Science, 248, 1112-1115.

Saraste, M., Sibbald, P. R. \& Wittinghofer, A. (1990). Trends Biochem. Sci. 15, 430-434.

Schlee, S., Groemping, Y., Herde, P., Seidel, R. \& Reinstein, J. (2001). J. Mol. Biol. 306, 889-899.

Schwefel, D., Fröhlich, C., Eichhorst, J., Wiesner, B., Behlke, J., Aravind, L. \& Daumke, O. (2010). Proc. Natl Acad. Sci. USA, 107, 20299-20304.

Seyffer, F., Kummer, E., Oguchi, Y., Winkler, J., Kumar, M., Zahn, R., Sourjik, V., Bukau, B. \& Mogk, A. (2012). Nature Struct. Mol. Biol. 19, 1347-1355.

Simonetta, K. R., Kazmirski, S. L., Goedken, E. R., Cantor, A. J., Kelch, B. A., McNally, R., Seyedin, S. N., Makino, D. L., O'Donnell, M. \& Kuriyan, J. (2009). Cell, 137, 659-671.

Stinson, B. M., Nager, A. R., Glynn, S. E., Schmitz, K. R., Baker, T. A. \& Sauer, R. T. (2013). Cell, 153, 628-639.

Tucker, P. A. \& Sallai, L. (2007). Curr. Opin. Struct. Biol. 17, 641-652.

Walker, J. E., Saraste, M., Runswick, M. J. \& Gay, N. J. (1982). EMBO J. 1, 945-951.

Wang, F., Mei, Z., Qi, Y., Yan, C., Hu, Q., Wang, J. \& Shi, Y. (2011). Nature (London), 471, 331-335.

Weibezahn, J., Tessarz, P., Schlieker, C., Zahn, R., Maglica, Z., Lee, S., Zentgraf, H., Weber-Ban, E. U., Dougan, D. A., Tsai, F. T., Mogk, A. \& Bukau, B. (2004). Cell, 119, 653-665.

Wendler, P., Ciniawsky, S., Kock, M. \& Kube, S. (2012). Biochim. Biophys. Acta, 1823, 2-14.

Werbeck, N. D., Kellner, J. N., Barends, T. R. M. \& Reinstein, J. (2009). Biochemistry, 48, 7240-7250.

Werbeck, N. D., Schlee, S. \& Reinstein, J. (2008). J. Mol. Biol. 378, 178-190.

Winkler, J., Tyedmers, J., Bukau, B. \& Mogk, A. (2012). J. Cell Biol. 198, 387-404.

Winn, M. D., Isupov, M. N. \& Murshudov, G. N. (2001). Acta Cryst. D57, 122-133.

Xia, D., Esser, L., Singh, S. K., Guo, F. \& Maurizi, M. R. (2004). J. Struct. Biol. 146, 166-179.

Zeymer, C., Werbeck, N. D., Schlichting, I. \& Reinstein, J. (2013). J. Biol. Chem. 288, 7065-7076.

Zhang, X. \& Wigley, D. B. (2008). Nature Struct. Mol. Biol. 15, $1223-$ 1227. 